\title{
SGLT2 Inhibitor and GLP-1 Receptor Agonist Combination Therapy Substantially Improved the Renal Function in a Patient with Type 2 Diabetes: Implications for Additive Renoprotective Effects of the Two Drug Classes
}

Kenta Nonomura $^{1}$, Katsumi Iizuka ${ }^{1,2}$, Yayoi Kuwabara-Ohmura ${ }^{1}$ and Daisuke Yabe $^{1}$

\begin{abstract}
:
A 72-year-old man had type 2 diabetes (T2D) that had been diagnosed at 54 years old. Macroalbuminuria was first detected at age 64. While his HbA1c had been kept below 7\%, his estimated glomerular filtration rate (eGFR) was declining rapidly. At 70 years old, his eGFR dropped below $50 \mathrm{~mL} / \mathrm{min} / 1.73 \mathrm{~m}^{2}$. A renal biopsy revealed diabetic nephropathy. Sodium glucose transporter 2 inhibitors (SGLT2i)/glucagon-like peptide-1 receptor agonists (GLP-1RA) combination therapy substantially improved his eGFR and urinary albumin level, and the renoprotective effect persisted for the two-year study period. These findings suggest that SGLT2i and GLP-1RA can additively improve the renal function in patients with T2D.
\end{abstract}

Key words: diabetic kidney disease, estimated glomerular filtration rate, type 2 diabetes mellitus, GLP-1 receptor agonist, SGLT2 inhibitor

(Intern Med 59: 1535-1539, 2020)

(DOI: 10.2169/internalmedicine.4323-19)

\section{Introduction}

Diabetes is the most frequent cause of end-stage renal disease (ESRD), which is defined by a glomerular filtration rate (GFR) $<15 \mathrm{~mL} / \mathrm{min} / 1.73 \mathrm{~m}^{2}$ ) (1). In ESRD, dialysis or a kidney transplant is required for the patient's survival. Therefore, the maintenance of appropriate glycemic control [HbAlc $<7.0 \%$ NGSP $(52 \mathrm{mmol} / \mathrm{mol}$ IFCC)] is highly recommended in order to prevent the development of ESRD in diabetes patients (2). Furthermore, it is widely accepted that a multidisciplinary approach targeting hypertension, dyslipidemia, and diabetes is beneficial for the prevention of ESRD in patients with diabetes and macroalbuminuria (2).

It was recently shown that the new anti-diabetic drugs the sodium glucose transporter 2 inhibitors (SGLT2is), including empagliflozin, canagliflozin and dapaglifrozin, as well as the glucagon-like peptide-1 receptor agonists (GLP-1RAs) liraglutide, semaglutide and dulaglutide not only suppress cardiovascular (CV) events but also prevent the development of
ESRD (3-8). The clinical benefits of SGLT2i/GLP-1 RA combination therapy on the renal function have therefore been receiving much attention lately $(9,10)$, although the effects remain to be evaluated in well-controlled clinical trials.

A progressive GFR decline precedes ESRD and has recently been established as the predominant clinical feature of diabetic kidney disease $(11,12)$. Because renal decline is linear in many patients, the Kidney Disease: Improving Global Outcomes (KDIGO) guidelines define rapid progression as a rate of eGFR decline $>5 \mathrm{~mL} / \mathrm{min} / \mathrm{year}$ (1). Thus, the definition of a rapid renal function decline is widely regarded as a decrease in the estimated GFR (eGFR), either in terms of the absolute rate of loss or percent change. While the SGLT2is empagliflozin, canagliflozin and dapagliflozin and the GLP-1RAs liraglutide, semaglutide and dulaglutide have been shown to prevent the development of ESRD, whether or not these drugs as monotherapy or in combination actually delay the rapid decline in the eGFR among patients with diabetes and macroalbuminuria is largely un-

${ }^{1}$ Department of Diabetes and Endocrinology, Graduate School of Medicine, Gifu University, Japan and ${ }^{2}$ Center for Nutritional Support and Infection Control, Gifu University Hospital, Japan

Received: December 10, 2019; Accepted: January 20, 2020; Advance Publication by J-STAGE: March 19, 2020

Correspondence to Dr. Katsumi Iizuka, kiizuka@gifu-u.ac.jp 


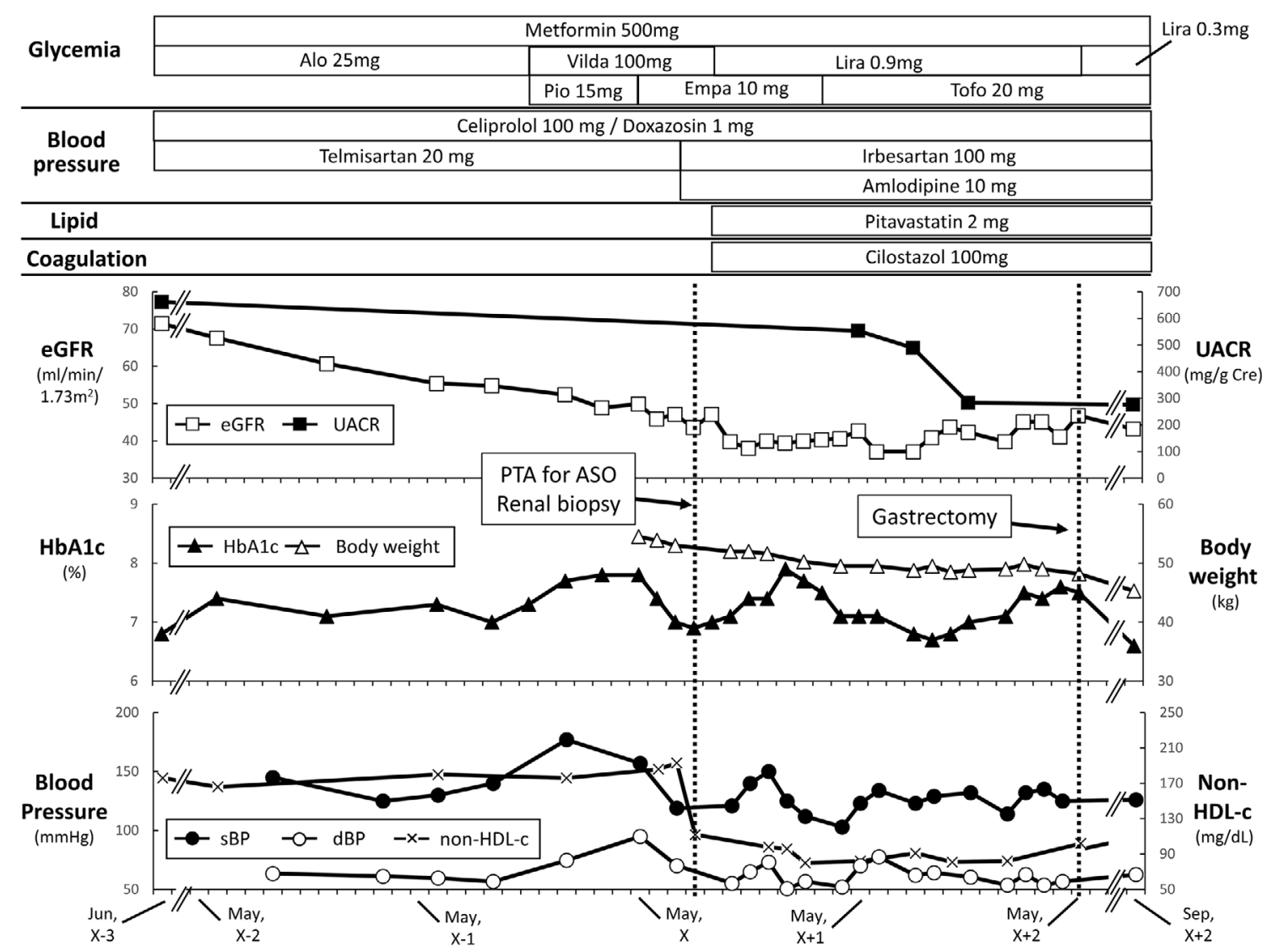

Figure 1. Clinical course of this case. The upper panel indicates the estimated glomerular filtration rate (eGFR, closed square) and urinary albumin creatinine ratio (UACR, open square). The middle panel indicates glycated hemoglobin A1c (HbA1c, open triangle) and body weight (body weight, closed triangle). The lower panel indicates the systolic blood pressure (sBP, open circle), diastolic blood pressure (dBP, closed circle), and non-HDL cholesterol (non-HDL-c, cross). The patient received percutaneous transluminal angioplasty (PTA) for arteriosclerosis obliterans (ASO) in August of year X, which likely decreased the eGFR. The patient received gastrectomy in May of year X+2 for his gastric cancer, which might have contributed to the body weight reduction. Alo: alogliptin, Empa: empagliflozin, Lira: liraglutide, Pio: pioglitazone, Tofo: tofoglifrozin, Vilda: vildagliptin

known.

\section{Case Report}

A 71-year-old man with type 2 diabetes (T2D) and arteriosclerosis obliterans (ASO) had been diagnosed with $\mathrm{T} 2 \mathrm{D}$ at an annual medical checkup at 54 years old, after which he commenced oral medication and then insulin therapy (total 30 units/day), although his HbA1c levels remained > $8.0 \%$ (IFCC, $63 \mathrm{mmol} / \mathrm{mol}$ IFCC). When he was referred to our hospital at 64 years old, he had an elevated HbA1c (NGSP, $8.0 \%$ and IFCC, $63 \mathrm{mmol} / \mathrm{mol}$ ) and macroalbuminuria [urinary albumin to creatinine ratio (UACR) $415 \mathrm{mg}$ / gCre] (Fig. 1).

He started $500 \mathrm{mg}$ of metformin once daily with dipeptidyl peptidase-4 inhibitors (DPP4is) (25 mg of alogliptin once daily later switched to $50 \mathrm{mg}$ of vildagliptin twice daily) or $15 \mathrm{mg}$ pioglitazone once daily; his glycemia was relatively well-controlled [HbA1c $6.0-8.0 \%$ (IFCC, 41-63 $\mathrm{mmol} / \mathrm{mol}$ )], but his eGFR started declining from year X-2 at a rate of $10.2 \mathrm{~mL} / \mathrm{min} / 1.73 \mathrm{~m}^{2}$ per year (Fig. 1). Along with the rapid decline in his eGFR, his UACR remained high ( $>500 \mathrm{mg} / \mathrm{gCre}$ ). Importantly, he showed no signs of neuropathy or retinopathy despite of the presence of the severe ASO.

In May of year X, he started receiving SGLT2is (10 mg of empagliflozin once daily later switched to $20 \mathrm{mg}$ of tofogliflozin) instead of pioglitazone to prevent a further decline in his eGFR. The change in the eGFR in the first month after SGLT2i initiation was $-48.0 \mathrm{~mL} / \mathrm{min} / 1.73 \mathrm{~m}^{2} /$ year, which was much higher than that of the eGFR from June of year X-2 to September of year X-1 (-10.2 $\mathrm{mL} / \mathrm{min} / 1.73 \mathrm{~m}^{2} /$ year $)$ (Period 1, 15 months) when he had been receiving DPP-4i and metformin. The change in the eGFR, along with decreases in his systolic blood pressure (sBP) and diastolic blood pressure $(\mathrm{dBP})$, suggested amelioration of his glomerular hyperfiltration upon initiation of SGLT2i.

In August of year X, percutaneous transluminal an- 


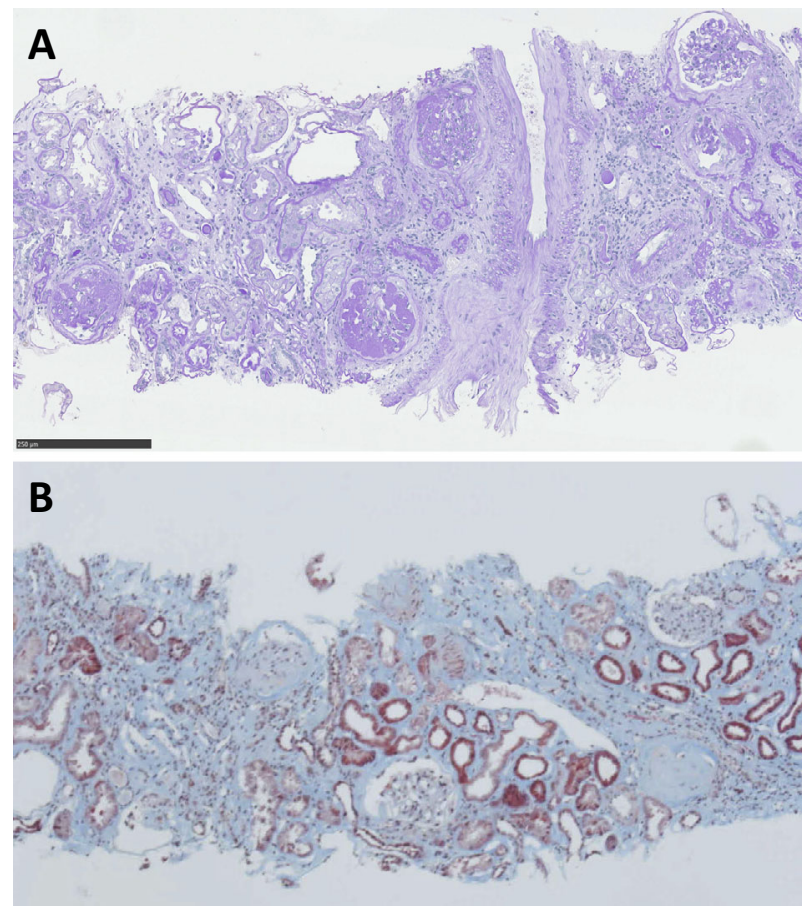

Figure 2. (A) Photograph of periodic acid-Schiffs (PAS) staining showing severe diffuse and global glomerulosclerosis of the remaining glomeruli and fibrin cap, glomerular solidification, and arteriolar hyalinosis and atherosclerosis, which is consistent with diabetic nephropathy. (B) Photograph of Masson's trichrome staining showing interstitial fibrosis, tubular atrophy, and tubulointerstitial cell infiltration. Scale bar $=250$ $\mu \mathrm{m}$.

gioplasty (PTA) for ASO and a renal biopsy were performed. Hematoxylin and Eosin, periodic acid-Schiff (PAS), Masson' trichrome, and periodic acid methenamine silver staining as well as immunofluorescence staining for light microscopy were performed on his biopsied samples. Of the 39 glomeruli examined, 13 were globally sclerotic. The remaining 26 showed diffuse increases in the amount of mesangial matrix and the number of mesangial cells, with some small mesangial nodules, hyaline vascular sclerosis, and a fibrin cap. In addition, interstitial fibrosis, interstitial edema, tubular atrophy, tubulointerstitial cell infiltration, and arteriolar hyalinosis and atherosclerosis were noted. These findings were compatible with a diagnosis of diabetic nephropathy and glomerulosclerosis (Fig. 2). Membranous nephropathy was ruled out by the results of immunofluorescent staining ( $\mathrm{IgG}, \mathrm{Ig} \mathrm{A}^{-}, \mathrm{IgM}^{+/}, \mathrm{C} 3, \mathrm{C}^{-}$and $\mathrm{C}^{-} \mathrm{q}^{-}$).

His eGFR declined substantially, likely due to the use of a contrast agent for endovascular treatment for his ASO in September of year X. However, the decline in the eGFR was blunted, and the UACR improved upon the initiation of SGLT2i (10 mg of empagliflozine or $20 \mathrm{mg}$ of tofogliflozine)/GLP-1RA (0.9 mg of liraglutide) combination therapy in addition to an angiotensin II receptor antagonist (ARB) (100 mg irbesartan), statin (pitavastatin $2 \mathrm{mg}$ once daily), and anti-platelet agent (cilostazol $100 \mathrm{mg}$ twice daily) (Fig. 1). The decline in the eGFR during Period 1 was $-10.2 \mathrm{~mL} / \mathrm{min} / 1.73 \mathrm{~m}^{2} /$ year, while that from October of year X to May of year X+2 (Period 2, 19 months) was +4.4 $\mathrm{mL} / \mathrm{min} / 1.73 \mathrm{~m}^{2} /$ year. The mean values of $\mathrm{sBP}[135.0 \pm 9.1$ $\mathrm{mmHg}$ (Period 1) and 126.5 $\pm 11.7 \mathrm{mmHg}$ (Period 2), $\mathrm{p}=$ 0.173], dBP [67.6 $\pm 9.2 \mathrm{mmHg}$ (Period 1) and 65.4 \pm 10.2 $\mathrm{mmHg}$ (Period 2), $\mathrm{p}=0.788$ ], and HbAlc [7.15\% $\pm 0.23 \%$ (Period 1) and $7.26 \% \pm 0.33 \%$ (Period 2), $\mathrm{p}=0.376$ ] were similar between the 2 periods, suggesting the glucose- and blood pressure-independent renoprotective effects of the combination therapy.

Despite gastrectomy for early-stage gastric cancer at 73 years old, the patient's renal function remained unchanged (Fig. 1). His body weight, height, and body mass index (BMI) in May of year X when he started SGLT2is were as follows: $54.5 \mathrm{~kg}, 148 \mathrm{~cm}$, and $24.9 \mathrm{~kg} / \mathrm{m}^{2}$, respectively. Changes in his bodyweight in addition to other clinical parameters, such as $\mathrm{HbA1c}, \mathrm{sBP}$, and $\mathrm{dBP}$, are indicated in Fig. 1.

\section{Discussion}

The current case showed a rapid decline in the renal function due to diabetic nephropathy as revealed by a renal biopsy and histological analysis. Importantly, SGLT2i/GLP1RA combination therapy, along with an ARB, statin and anti-platelet agent, substantially suppressed the progression of renal impairment, consistent with recent results of cardiovascular outcome trials and relevant clinical trials using SGLT2i or GLP-1RA (3-8, 13).

Preclinical studies have suggested that GLP-1RAs reduce inflammation and oxidative stress, thereby preventing diabetic nephropathy and acute kidney injury (14-17). Indeed, the eGFR decline was found to be approximately $2 \mathrm{~mL} / \mathrm{min} /$ $1.73 \mathrm{~m}^{2}$ per year in T2D patients with a baseline eGFR of $30-59 \mathrm{~mL} / \mathrm{min} / 1.73 \mathrm{~m}^{2}$ who were treated by GLP-1RA liraglutide, while it was approximately $4 \mathrm{~mL} / \mathrm{min} / 1.73 \mathrm{~m}^{2}$ per year in a placebo group (estimated trial-group ratio in favor of liraglutide, 1.07; 95\% confidence interval, 1.04-1.10; $\mathrm{p}<$ 0.001) (6). SGLT2is favorably affect body weight, BP, serum uric acid, and glomerular hyperfiltration (13). The recognized renoprotective effects of SGLT2is include the suppression of hyperplasia/hypertrophy, inflammation, and fibrosis in the proximal tubular cells, utilization of ketone bodies, restored tubuloglomerular feedback, decreased oxygen consumption, improvement in anemia, and preconditioning against ischemia/reperfusion $(18,19)$. In obese diabetic mice, ipragliflozin reduced renal cortical hypoxia and abnormal hemodynamics, resulting in protection against early diabetic nephropathy (20). Furthermore, dapagliflozin attenuates renal tubulointerstitial fibrosis by regulating signal transducer and activator of transcription (STAT)1/transforming growth factor (TGF) $\beta 1$ signaling in streptozotocin (STZ)-induced type 1 diabetic mice (21). It was also clinically demonstrated that SGLT2is are particularly effective in preventing the eGFR decline in T2D patients with a baseline eGFR of $60-89$ or $<60 \mathrm{~mL} / \mathrm{min} / 1.73 \mathrm{~m}^{2}$ compared to those 
with a baseline eGFR of $>90 \mathrm{~mL} / \mathrm{min} / 1.73 \mathrm{~m}^{2}(13)$. The eGFR decline in our patient was initially $10.2 \mathrm{~mL} / \mathrm{min} / 1.73$ $\mathrm{m}^{2}$ per year in the most recent 2 years before the initiation of SGLT2i/GLP-1RA combination therapy, and there was almost no decline in the 2 years after the initiation of the therapy.

While magnetic resonance imaging dependent on the levels of inflammatory biomarkers such as urinary 8hydroxydeoxyguanosin $(8-\mathrm{OHdG})$ and hypoxia markers such as blood oxygen was not performed before or after the initiation of SGLT2i/GLP-1RA combination therapy, our current findings suggest that SGLT2is and GLP-1RAs cooperatively prevent the eGFR decline. Whether or not the renoprotective effects of SGLT2is and GLP-1RAs are additive should be investigated in well-controlled clinical trials.

The progression of glomerular, exudative, tubulointerstitial, and vascular lesions is known to be associated with renal death (22). Furthermore, interstitial fibrosis, tubular atrophy, and tubulointerstitial inflammation were found to be strongly associated with the renal survival $(22,23)$. In the histology of our patient, interstitial fibrosis, tubular atrophy and tubulointerstitial inflammation were observed, suggesting a poor renal prognosis. It would therefore be interesting to compare the histological changes before and after the initiation of SGLT2i/GLP-1RA combination therapy in order to better understand why the decline in the eGFR was delayed after the initiation of therapy in the current case.

Multidisciplinary treatment using glucose-lowering agents, lipid-lowering drugs, and anti-platelet agents as well as blood pressure-lowering agents, especially ARBs and angiotensin-converting-enzyme inhibitors, has been shown to be effective for preventing diabetic nephropathy $(24,25)$. In the present case, since the $\mathrm{sBP}, \mathrm{dBP}$, and $\mathrm{HbA} 1 \mathrm{c}$ did not differ significantly after the initiation of SGLT2i and GLP1RA, the combination therapy in addition to ARB, statin, and anti-platelet agents may contribute to the maintenance of the renal function in a glucose- and blood pressureindependent manner. It is also possible that the patient changed his lifestyle after his hospitalization for the renal biopsy, which might have helped delay the rapid decline in his eGFR.

In conclusion, we encountered a case in which SGLT2i/ GLP-1RA combination therapy substantially improved renal parameters, such as the eGFR and urinary albumin. The findings in the current case suggest that SGLT2i and GLP1RA cooperatively improve the renal function through different mechanisms.

All procedures performed in studies involving human participants were in accordance with the 1964 Declaration of Helsinki and its later amendments or comparable ethical standards. Informed consent was obtained from all individual participants included in the study.

Author's disclosure of potential Conflicts of Interest (COI). Katsumi Iizuka: Honoraria, Novo Nordisk Pharma and Kowa.
Daisuke Yabe: Honoraria, MSD, Novo Nordisk Pharma and Taisho Toyama Pharmaceutical; Research funding, Nippon Boehringer Ingelheim, Eli Lilly, Taisho Toyama Pharmaceutical, MSD, Ono Pharmaceutical, Novo Nordisk Pharma, Arklay and Takeda Pharmaceutical.

\section{Financial Support}

This work was supported by grants from the Japan Society for the Promotion of Sciences (JSPS) [KAKENHI Grant 17K09825 (to D.Y.) and 17K00850 (to K.I)].

\section{Acknowledgement}

The authors are very grateful to the patient for his kind contribution to this study. The authors also thank J. Kawada and M. Kato for their technical assistance, and M. Yato, Y. Ogiso and M. Nozu for their secretarial assistance.

\section{References}

1. Chapter 2: Definition, identification, and prediction of CKD progression. Kidney Int Suppl 3(Suppl 2011): 63-72, 2013.

2. Gaede P, Vedel P, Larsen N, Jensen GV, Parving HH, Pedersen O. Multifactorial intervention and cardiovascular disease in patients with type 2 diabetes. N Engl J Med 348: 383-393, 2003.

3. Wanner C, Inzucchi SE, Lachin JM, et al.; EMPA-REG OUTCOME Investigators. Empagliflozin and progression of kidney disease in type 2 diabetes. N Engl J Med 375: 323-334, 2016.

4. Neal B, Perkovic V, Mahaffey KW, et al.; CANVAS Program Collaborative Group. Canagliflozin and cardiovascular and renal events in type 2 diabetes. N Engl J Med 377: 644-657, 2017.

5. Marso SP, Bain SC, Consoli A, et al.; SUSTAIN-6 Investigators. Semaglutide and cardiovascular outcomes in patients with type 2 diabetes. N Engl J Med 375: 1834-1844, 2016.

6. Mann JFE, Ørsted DD, Brown-Frandsen K, et al.; LEADER Steering Committee and Investigators. Liraglutide and renal outcomes in type 2 diabetes. N Engl J Med 377: 839-848, 2017.

7. Wiviott SD, Raz I, Bonaca MP, et al.; DECLARE-TIMI 58 Investigators. Dapagliflozin and cardiovascular outcomes in type 2 diabetes. N Engl J Med 380: 347-357, 2019.

8. Gerstein HC, Colhoun HM, Dagenais GR, et al.; REWIND Investigators. Dulaglutide and renaloutcomes in type 2 diabetes: an exploratory analysis of the REWIND randomised, placebo-controlled trial. Lancet 394: 131-138, 2019.

9. DeFronzo RA. Combination therapy with GLP-1 receptor agonist and SGLT2 inhibitor. Diabetes Obes Metab 19: 1353-1362, 2017.

10. Nagahisa T, Saisho Y. Cardiorenal protection: potential of SGLT2 inhibitors and GLP-1 receptor agonists in the treatment of type 2 Diabetes. Diabetes Ther 10: 1733-1752, 2019.

11. Krolewski AS, Skupien J, Rossing P, Warram JH. Fast renal decline to end-stage renal disease: an unrecognized feature of nephropathy in diabetes. Kidney Int 91: 1300-1311, 2017.

12. Krolewski AS. Progressive renal decline: the new paradigm of diabetic nephropathy in type 1 diabetes. Diabetes Care 38: 954-962, 2015.

13. Zelniker TA, Wiviott SD, Raz I, et al. SGLT2 inhibitors for primary and secondary prevention of cardiovascular and renal outcomes in type 2 diabetes: a systematic review and meta-analysis of cardiovascular outcome trials. Lancet 393: 31-39, 2019.

14. Kodera R, Shikata K, Kataoka HU, et al. Glucagon-like peptide-1 receptor agonist ameliorates renal injury through its antiinflammatory action without lowering blood glucose level in a rat model of type 1 diabetes. Diabetologia 54: 965-978, 2011.

15. Fujita H, Morii T, Fujishima H, et al. The protective roles of GLP$1 \mathrm{R}$ signaling in diabetic nephropathy: possible mechanism and therapeutic potential. Kidney Int 85: 579-589, 2014. 
16. von Scholten BJ, Persson F, Rosenlund S, et al. Effects of liraglutide on cardiovascular risk biomarkers in patients with type 2 diabetes and albuminuria: a sub-analysis of a randomized, placebocontrolled, double-blind, crossover trial. Diabetes Obes Metab 19: 901-905, 2017.

17. Seino Y, Yabe D. Glucose-dependent insulinotropic polypeptide and glucagon-like peptide-1: incretin actions beyond the pancreas. J Diabetes Investig 4: 108-130, 2013.

18. Hirakawa Y, Tanaka T, Nangaku M. Renal hypoxia in CKD; pathophysiology and detecting methods. Front Physiol 8: 99 2017.

19. Ito $M$, Tanaka $\mathrm{T}$. The anticipated renoprotective effects of sodiumglucose cotransporter 2 inhibitors. Intern Med 57: 2105-2114, 2018 .

20. Kamezaki M, Kusaba T, Komaki K, et al. Comprehensive renoprotective effects of ipragliflozin on early diabetic nephropathy in mice. Sci Rep 8: 4029, 2018

21. Huang F, Zhao Y, Wang Q, et al. Dapagliflozin attenuates renal tubulointerstitial fibrosis associated with type 1 diabetes by regulating STAT1/TGFß1 signaling. Front Endocrinol (Lausanne) 10: 441, 2019.
22. Mise $\mathrm{K}$, Hoshino $\mathrm{J}$, Ubara $\mathrm{Y}$, et al. Renal prognosis a long time after renal biopsy on patients with diabetic nephropathy. Nephrol Dial Transplant 29: 109-118, 2014.

23. Okada T, Nagao T, Matsumoto $H$, et al. Histological predictors for renal prognosis in diabetic nephropathy in diabetes mellitus type 2 patients with overt proteinuria. Nephrology (Carlton) 17: 68-75, 2012

24. Gaede P, Lund-Andersen $H$, Parving $\mathrm{HH}$, Pedersen O. Effect of a multifactorial intervention on mortality in type 2 diabetes. $\mathrm{N}$ Engl J Med 358: 580-591, 2008.

25. Ueki K, Sasako T, Okazaki Y, et al.; J-DOIT3 Study Group. Effect of an intensified multifactorialintervention on cardiovascular outcomes and mortality in type 2 diabetes (J-DOIT3): an open-label, randomised controlled trial. Lancet Diabetes Endocrinol 5: 951964, 2017.

The Internal Medicine is an Open Access journal distributed under the Creative Commons Attribution-NonCommercial-NoDerivatives 4.0 International License. To view the details of this license, please visit (https://creativecommons.org/licenses/ by-nc-nd/4.0/).

(C) 2020 The Japanese Society of Internal Medicine Intern Med 59: 1535-1539, 2020 